\title{
Control of vortex pair states by post-deposition interlayer exchange coupling modification
}

\author{
Sebastian Wintz, ${ }^{1, *}$ Thomas Strache, ${ }^{1, \dagger}$ Michael Körner, ${ }^{1}$ Christopher Bunce, ${ }^{1, \ddagger}$ Anja Banholzer, ${ }^{1}$ Ingolf Mönch, ${ }^{2}$ \\ Roland Mattheis, ${ }^{3}$ Jörg Raabe, ${ }^{4}$ Christoph Quitmann, ${ }^{4}$ Jeffrey McCord, ${ }^{1,}{ }^{\S}$ Artur Erbe, ${ }^{1}$ Kilian Lenz, ${ }^{1}$ and Jürgen Fassbender ${ }^{1}$ \\ ${ }^{1}$ Helmholtz-Zentrum Dresden-Rossendorf, 01328 Dresden, Germany \\ ${ }^{2}$ Institute for Integrative Nanosciences, IFW Dresden, 01069 Dresden, Germany \\ ${ }^{3}$ Institut für Photonische Technologien, 07702 Jena, Germany \\ ${ }^{4}$ Swiss Light Source, Paul Scherrer Institut, 5232 Villigen, Switzerland
}

(Received 27 January 2012; revised manuscript received 22 March 2012; published 9 April 2012)

\begin{abstract}
We report on both the global and micromagnetic properties of interlayer exchange coupled spin systems. Irradiation with $\mathrm{Ne}$ ions is employed to achieve a phase transition from antiferromagnetic to ferromagnetic coupling. For extended trilayer films a full quantitative analysis of the bilinear and biquadratic coupling constants is performed. With increasing ion fluence we observe a steady increase of the bilinear coupling constant at an almost negligible decrease in saturation magnetization. The mixing of atoms at the layer interfaces is identified as the origin for this. The effects of ion modification on the magnetic microstructure are studied for the model system of layered vortex pairs. X-ray microscopy is used to directly image the individual magnetization circulations in trilayer disks. The circulation configuration is found to be determined by the film coupling for both coupling orientations with a homogenous coupling angle throughout the structure. For the vortex cores, however, micromagnetic simulations indicate that due to the significant local demagnetization fields, parallel states are always energetically preferred. Nevertheless antiparallel configurations are metastable, having their signature in reduced core diameters. Our study provides new results on spin structures in interlayer exchange coupled trilayers and it demonstrates a promising way to control the local interlayer coupling post-deposition.
\end{abstract}

DOI: 10.1103/PhysRevB.85.134417

PACS number(s): 75.70.Kw, 81.40.Rs, 75.75.-c, 68.37.Yz

\section{INTRODUCTION}

Magnetic multilayers have been the key to fundamental effects such as giant magneto resistance ${ }^{1,2}$ or interlayer exchange coupling (IEC) $)^{3,4}$ that are nowadays of far-reaching technological importance. IEC describes the magnetic coupling of two ferromagnetic layers via a thin nonmagnetic interlayer. This interaction arises from an induced spin polarization of the interlayer's electrons..$^{5}$ Depending on the interlayer material and thickness, IEC typically causes an energetic preference for either a parallel or an antiparallel orientation of the two ferromagnetic layers, which is referred to as ferromagnetic (fm) or antiferromagnetic (afm) coupling, respectively. ${ }^{6}$ This is described empirically by a positive or negative bilinear coupling constant $J_{\mathrm{L}}$ in the expression for the IEC areal energy density

$$
\sigma_{\mathrm{IEC}}=-J_{\mathrm{L}} \frac{\mathbf{M}_{1} \cdot \mathbf{M}_{2}}{M_{1} M_{2}}-J_{\mathrm{Q}} \frac{\left(\mathbf{M}_{1} \cdot \mathbf{M}_{2}\right)^{2}}{\left(M_{1} M_{2}\right)^{2}},
$$

where $\mathbf{M}_{i}$ is the magnetization vector of the $i$ th ferromagnetic layer. ${ }^{7}$ The additional biquadratic coupling constant $\left(J_{\mathrm{Q}}\right)$ may induce an energetic preference for a $90^{\circ}$ coupling orientation ${ }^{7,8}$ that is mainly attributed to thickness fluctuations. ${ }^{9}$ For $J_{\mathrm{L}}$, a damped sinusoidal dependence on the interlayer thickness $(t)$ was discovered, ${ }^{10}$ which yields a way to set the IEC at the time of the layer deposition. Based on the modification of magnetic properties by ion irradiation, ${ }^{11,12}$ Demokritov et al. ${ }^{13}$ pioneered a promising post-deposition way to change the IEC in $\mathrm{Fe} / \mathrm{Cr} / \mathrm{Fe}$ continuous films from afm to fm by applying low fluences of He ions. A similar approach has been made for trilayers containing a $\mathrm{Ru}$ interlayer ${ }^{14}$ and also a local modification of IEC has been reported. ${ }^{15-18}$

While most investigations have addressed IEC in continuous films, so far there is little known about its effect on the magnetic microstructure, i.e., the local magnetization distribution in the different layers. ${ }^{19-21}$ Only recently it was shown that IEC can be used to control the relative circulation configuration of interlayer coupled vortex pairs. ${ }^{22,23}$ As sketched in Fig. 1 such a micromagnetic vortex consists of a planar, flux-closing magnetization curl, that turns out of the plane in the small central core region. ${ }^{19,24-28}$ Both, the curl's rotation sense (circulation $c$ ) and the core orientation (polarity $p$ ) can be in either of two states $(+1,-1)$. Thus, for a pair of two layered vortices, the relative circulation orientation $C=\prod_{i} c_{i}$ generally can be either ferromagnetic (FM) or antiferromagnetic (AF) while the core alignment $P=\prod_{i} p_{i}$ can be either parallel (PL) or antiparallel (AP). ${ }^{22,29-31}$

A remaining question is whether for nonuniform spin distributions in polycrystalline films the IEC is homogenous on a sub-microscopic scale, and whether this would necessarily result in a constant coupling angle. In particular, the effects of the ion-based IEC modification on the magnetic microstructure are unknown. Therefore, in this work we will report our findings on the consequences of ion irradiation for both the global and the local properties of IEC. We will provide a quantitative analysis of the ion-induced modification of the global IEC constants $J_{l}$ and $J_{q}$ along with an explanation of the relevant underlying processes. The local magnetic microstructure of nonirradiated and irradiated IEC stacks is investigated for the nonuniform model system of layered vortex pairs. Special emphasis is placed on the different coupling schemes for $C$ and $P$.

\section{EXPERIMENTAL DETAILS}

\section{A. Sample fabrication}

For investigating the effect of ion irradiation on the IEC we have prepared two different functional magnetic trilayer 


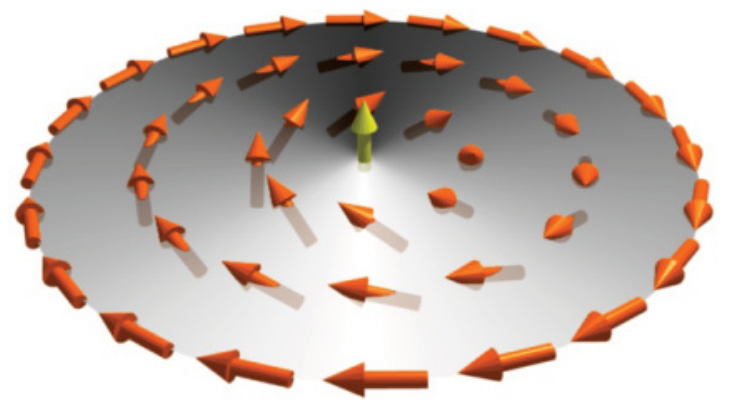

FIG. 1. (Color online) Spin vortex in a single-layer disk with arrows indicating the magnetization direction. Two states are possible for the sense of the planar magnetization curl (circulation, $c$ ) as well as for the orientation of the central core (polarity, $p$ ), giving rise to a binary handedness of the system.

films along with two single-layer reference films which are specified in Table I. The films were deposited by dc magnetron sputtering in a ten-target ultrahigh vacuum system. The macroscopic coupling parameters have been studied using continuous films, whereas the specific magnetic microstructure has been obtained from vortex pair states in patterned trilayer elements.

Both functional trilayer films consist of two ferromagnetic layers that are separated by a nonmagnetic spacer. They were deposited either onto thermally oxidized Si chips or x-raytransparent $\mathrm{SiN}$ membranes with a thickness of $t=100 \mathrm{~nm}$ (see Fig. 2). Aluminum capping layers prevent the stacks from oxidation. Co and Permalloy $\left(\mathrm{Ni}_{81} \mathrm{Fe}_{19}\right)$ have been chosen for the ferromagnetic layers in order to allow for a layer-selective detection by $\mathrm{x}$-ray techniques. The different spacer materials and thicknesses are expected to provide a strong IEC on the one hand $(\mathrm{Ru}: t \approx 0.8 \mathrm{~nm}),{ }^{4}$ and a pure dipolar coupling (PDC) with no IEC contribution on the other hand (Cu: $t=10 \mathrm{~nm})$. The nominal thicknesses of the ferromagnetic layers $(t=$ $25 \mathrm{~nm}$ ) are in the range for which a vortex is expected as the ground state of a single-layer micron-sized element. ${ }^{32-34}$

The post-deposition modification of the IEC was achieved by irradiating $\mathrm{Co} / \mathrm{Ru} / \mathrm{NiFe}(25)$ films with $\mathrm{Ne}^{+}$from an ion implanter. $\mathrm{Ne}^{+}$fluences between $10^{13} \mathrm{~cm}^{-2}$ and $2 \times 10^{15} \mathrm{~cm}^{-2}$ (see Table II) have been used. Based on simulations, ${ }^{35}$ a kinetic ion energy of $60 \mathrm{keV}$ has been selected to match the mean penetration depth with the position of the Ru interlayer. The morphological effect of the ion bombardment has been visualized by transmission electron microscopy (TEM) and has been simulated using the SRIM ${ }^{35}$ and TRIDYN ${ }^{36}$ software packages.

TABLE I. Sample naming and composition. The thickness of the ferromagnetic layers was measured by TEM, whereas the thicknesses of the other layers are nominal. Si* corresponds to the position of the substrate ( $\mathrm{SiN}$ or oxidized $\mathrm{Si}$ ).

\begin{tabular}{lc}
\hline \hline Sample & Composition $[t(\mathrm{~nm})]$ \\
\hline $\mathrm{Co}(25)$ & $\mathrm{Si}^{*} / \mathrm{Co}(23.9) / \mathrm{Al}(5)$ \\
$\mathrm{NiFe}(25)$ & $\mathrm{Si}^{*} / \mathrm{Ni}_{81} \mathrm{Fe}_{19}(21.4) / \mathrm{Al}(5)$ \\
$\mathrm{Co} / \mathrm{Ru} / \mathrm{NiFe}(25)$ & $\mathrm{Si} / \mathrm{Co}(23.9) / \mathrm{Ru}(0.8) / \mathrm{Ni}_{81} \mathrm{Fe}_{19}(21.4) / \mathrm{Al}(5)$ \\
$\mathrm{Co} / \mathrm{Cu} / \mathrm{NiFe}(50)$ & $\mathrm{Si} / \mathrm{Co}(47.8) / \mathrm{Cu}(10) / \mathrm{Ni}_{81} \mathrm{Fe}_{19}(42.8) / \mathrm{Al}(5)$ \\
\hline \hline
\end{tabular}

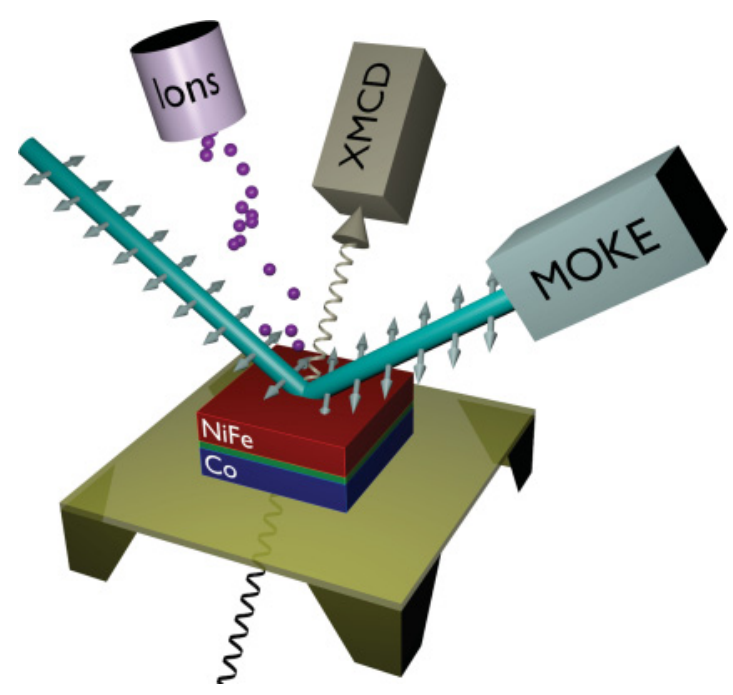

FIG. 2. (Color online) Sketch of the sample geometry and applied experimental techniques. Patterned trilayer element, consisting of two ferromagnetic layers $(\mathrm{Co}, \mathrm{NiFe})$ and a nonmagnetic interlayer on an $\mathrm{x}$-ray-transparent $\mathrm{SiN}$ substrate. Irradiation with $\mathrm{Ne}$ ions is used to modify the IEC between the ferromagnetic layers. The magnetization reversal (of continuous trilayer films) is detected by MOKE and SQUID. XMCD is used to image the magnetic structure with layerselective lateral resolution.

Circular disks and square-shaped elements (squares) with a base size of $2 \mu \mathrm{m}$ have been fabricated in order to confine vortices into IEC trilayer elements. For this, electronbeam lithography was performed in combination with liftoff processing. Additionally, a $\mathrm{Cu}$ stripline of $t=150 \mathrm{~nm}$ was attached to these samples as an excitation source for further studies.

\section{B. Magnetometry}

The magnetic properties and the coupling parameters of the continuous films were obtained from different magnetometry methods: Inductive measurements [superconducting quantum interference device (SQUID) and BH looper], magneto-optical Kerr effect (MOKE), and ferromagnetic resonance (FMR).

The inductive techniques measure the projection of the sample's total magnetic moment onto an axis of sensitivity, whereas MOKE gives a more surface-sensitive signal due to the finite penetration depth of light. Both methods were used to record magnetization reversal curves. From these curves, the

TABLE II. Bilinear $\left(J_{\mathrm{L}}\right)$ and biquadratic $\left(J_{\mathrm{Q}}\right)$ IEC coupling constants for the $\mathrm{Co} / \mathrm{Ru} / \mathrm{NiFe}(25)$ stack as a function of $\mathrm{Ne}^{+}$ irradiation at $60 \mathrm{keV}$.

\begin{tabular}{lcc}
\hline \hline $\mathrm{Ne}\left(10^{13} \mathrm{~cm}^{-2}\right)$ & $J_{\mathrm{L}}\left(\mathrm{mJ} / \mathrm{m}^{2}\right)$ & $J_{\mathrm{Q}}\left(\mathrm{mJ} / \mathrm{m}^{2}\right)$ \\
\hline 0 & -0.35 & -0.07 \\
1 & -0.33 & -0.05 \\
5 & -0.27 & -0.04 \\
10 & -0.20 & -0.03 \\
20 & -0.13 & -0.01 \\
30 & -0.05 & -0.02 \\
200 & 0.7 & 0 \\
\hline \hline
\end{tabular}


characteristic coercive field $\left(H_{\mathrm{c}}\right)$, the saturation field $\left(H_{\mathrm{s}}\right)$, and if applicable also the IEC coupling field $\left(H_{\mathrm{cp}}\right)$ were obtained. The saturation magnetization $\left(M_{\mathrm{s}}\right)$ values of the single-layer films were deduced from the perpendicular $H_{\mathrm{s}}$, neglecting intrinsic perpendicular anisotropy contributions. ${ }^{37}$ A planar hard axis magnetization reversal loop was used to estimate the intrinsic uniaxial in-plane anisotropy constant $\left(K_{\mathrm{u}}\right)$ via $K_{\mathrm{u}}=\mu_{0} H_{\mathrm{k}} M_{\mathrm{s}} / 2$. The equilibrium orientation between the two ferromagnetic layers of the trilayer films was derived from the relative magnetic moment at remanence $\mu_{\mathrm{r}} / \mu_{\mathrm{s}}$. Quantitative values for the afm coupling parameters $\left(J_{\mathrm{L}}, J_{\mathrm{Q}}\right)$ were obtained by fitting the experimental curves with an energy-minimized modified Stoner-Wohlfarth model ${ }^{19}$ for a planar magnetization reversal that in addition to the Zeeman and anisotropy energies also accounts for $\sigma_{\mathrm{IEC}}$,

$$
\begin{aligned}
\sigma\left(H, \phi_{1}, \phi_{2}\right)= & -J_{\mathrm{L}} \cos \left(\phi_{1}-\phi_{2}\right)-J_{\mathrm{Q}} \cos ^{2}\left(\phi_{1}-\phi_{2}\right) \\
& -\sum_{i=1}^{2}\left(\mu_{0} H M_{i} t_{i} \cos \phi_{i}+K_{u, i} t_{i} \cos ^{2} \phi_{i}\right),
\end{aligned}
$$

where $\phi_{i}$ is the azimuthal magnetization angle of the $i$ th layer with respect to the external field $H$. The easy axes are assumed to be parallel.

FMR measurements were performed to analyze the IEC for the fm case. A broadband vector network analyzer based FMR setup was used with the samples placed upside down on a coplanar waveguide. The resonances were recorded by sweeping the external magnetic field at a fixed microwave frequency of $\approx 14 \mathrm{GHz}$. For the single-layer $\mathrm{Co}$ and $\mathrm{NiFe}$ samples one (the uniform) resonance was observed. For the coupled trilayer samples two resonances (the optical and the acoustic mode) were detected. The coupling constants were obtained by fitting the appropriate resonance equation to the polar-angle-dependent FMR data (see Ref. 38). A review on FMR on coupled trilayers can be found in Ref. 39.

\section{Magnetic x-ray microscopy}

Soft $\mathrm{x}$-ray magnetic microscopy ${ }^{40,41}$ was performed in order to image the local magnetic orientation of the coupled trilayer structures. The scanning transmission $\mathrm{x}$-ray microscopy (STXM) setup used allows for an element-selective detection of the magnetization orientation with a lateral resolution below $25 \mathrm{~nm} .{ }^{42,43}$ While a monochromatic $\mathrm{x}$-ray beam is focused onto the sample by a Fresnel zone plate, the sample is scanned through the beam and the transmitted intensity yields the 2D image. Magnetic contrast stems from x-ray magnetic circular dichroism (XMCD) that occurs for circularly polarized $x$ rays at the L-absorption edges. ${ }^{44}$ The normalized difference of images taken at the two opposing circular polarizations leads to a pure magnetic contrast. The XMCD absorption coefficient is then proportional to the projection of the sample's magnetization orientation $\mathbf{m}=\mathbf{M} / M$ onto the photon propagation direction. In order to sense an in-plane magnetization component, the sample's normal is tilted by $30^{\circ}$ with respect to the beam axis (see Fig. 2). Layer-selective magnetic information is gained by imaging the sample with two different photon energies corresponding to the $\mathrm{Fe} \mathrm{L}_{3}$ absorption edge $(\approx 707 \mathrm{eV})$ and to the $\mathrm{Co} \mathrm{L}_{3}$-absorption edge $(\approx 778 \mathrm{eV})$, respectively, since only the layer that contains the specific element contributes to the magnetic signal.

\section{RESULTS AND DISCUSSION}

\section{A. IEC modification in continuous films}

Continuous films were used to characterize the IEC and to obtain quantitative values for the magnetic properties and the coupling parameters. For the single-layer reference films the following magnetic parameters were derived:

$$
\begin{aligned}
M_{\mathrm{s}}^{\mathrm{Co}} & =1.27 \times 10^{6} \mathrm{~A} / \mathrm{m}, \quad K_{\mathrm{u}}^{\mathrm{Co}}=0.8 \mathrm{~kJ} / \mathrm{m}^{3}, \\
M_{\mathrm{s}}^{\mathrm{NiFe}} & =0.74 \times 10^{6} \mathrm{~A} / \mathrm{m}, \quad K_{\mathrm{u}}^{\mathrm{NiFe}}=0.2 \mathrm{~kJ} / \mathrm{m}^{3} .
\end{aligned}
$$

Figure 3 shows the change of the easy axis hysteresis curve upon $\mathrm{Ne}^{+}$irradiation and the transition from afm IEC to fm IEC. The as-deposited sample [solid curve in Fig. 3(a)] shows the typical behavior of an afm coupled system. ${ }^{22}$ At a saturation field of $\mu_{0} H_{\mathrm{s}} \approx 45 \mathrm{mT}$ both layers are aligned parallel to the external field. When the field is reduced, the IEC forces a continuous reorientation of the $\mathrm{NiFe}$ layer, resulting in an antiparallel alignment of $\mathrm{Co}$ and $\mathrm{NiFe}$ at $\mu_{0} H_{\mathrm{cp}} \approx 10 \mathrm{mT}$. During this process, also the Co layer slightly changes its orientation (effect not noticeable here), though at $10 \mathrm{mT}$ it again points parallel to the external field. The presumption of an antiparallel orientation in the field range $(-10 \cdots 10)$ $\mathrm{mT}$ is supported by the measured relative magnetic moment ${ }^{22}$ of $\mu_{r} / \mu_{s}=0.33$, which fits to the expected value. At a field of $\mu_{0} H \approx-2.5 \mathrm{mT}$ the system performs a collective $180^{\circ}$

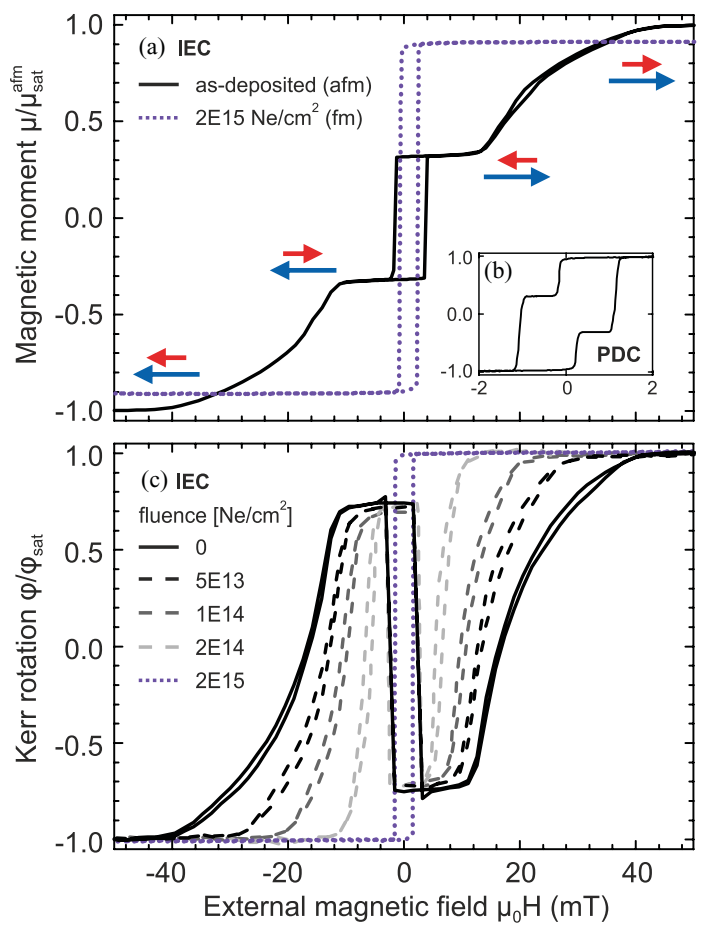

FIG. 3. (Color online) Normalized hysteresis curves of continuous trilayer films irradiated with different $\mathrm{Ne}^{+}$fluences. (a) SQUID loop of $\mathrm{Co} / \mathrm{Ru} / \mathrm{NiFe}(25)$. (b) $\mathrm{BH}$ loop of the PDC film $\mathrm{Co} / \mathrm{Cu} / \mathrm{NiFe}(50)$. (c) $\mathrm{MOKE}$ loop of $\mathrm{Co} / \mathrm{Ru} / \mathrm{NiFe}(25)$, which predominantly provides the $\mathrm{NiFe}$ signal. Arrows indicate the $\mathrm{Co}$ (blue) and $\mathrm{NiFe}$ (red) magnetization orientation. 
switching in order to bring the Co-dominated net moment parallel to the external field. Further increasing the negative field leads again to a $\mathrm{NiFe}$ rotation and eventually to saturation. Note that the reversal of continuous films typically involves the formation of magnetic domains with different orientations. However the reversal scheme described is representative for the individual domains.

If the same sample is exposed to a fluence of $2 \times 10^{15} \mathrm{~cm}^{-2}$ $\mathrm{Ne}$ ions at $60 \mathrm{keV}$, its reversal behavior changes drastically. Instead of the typical afm IEC curve, the system now exhibits an fm coupled reversal [dotted curve in Fig. 3(a)]. However, the coercivity of $\mu_{0} H_{\mathrm{c}} \approx 1.5 \mathrm{mT}$ is comparable to the switching field of the unirradiated sample. The magnetic moment at saturation is normalized to that of the as-deposited sample. The irradiation causes an average reduction of the saturation magnetization to $91 \%$ of the original value. In summary, the IEC in the $\mathrm{Co} / \mathrm{Ru} / \mathrm{NiFe}(25)$ system can be reversed from afm to fm by $\mathrm{Ne}^{+}$irradiation with a fluence of $2 \times 10^{15} \mathrm{~cm}^{-2}$ without significantly altering the magnetic properties of the individual layers.

As a reference for PDC behavior we show the reversal of the $\mathrm{Co} / \mathrm{Cu} / \mathrm{NiFe}(50)$ stack in Fig. 3(b), measured by a BH looper as already shown in Ref 22. Starting from a parallel orientation of both layers at negative saturation, the reversal occurs via two distinct switching steps at $\mu_{0} H \approx 0.25 \mathrm{mT}(\mathrm{NiFe})$ and $\mu_{0} H \approx 1.0 \mathrm{mT}(\mathrm{Co})$, respectively. This means that for such a PDC system both layers can be aligned either parallel or antiparallel at small external fields and even at remanence (minor loop; not shown).

In order to study the details of the irradiation-induced transition from IEC afm to IEC fm, continuous films exposed to different $\mathrm{Ne}^{+}$fluences between 0 and $2 \times 10^{15} \mathrm{~cm}^{-2}$ were investigated. Figure 3(c) shows the hysteresis curves for five fluences measured by MOKE. As MOKE is a surfacesensitive method, most of the recorded signal originates from the $\mathrm{NiFe}$ layer. This gives rise to the different shape of the MOKE curves with respect to the equivalent SQUID measurements [see Fig. 3(a)] for the as-deposited afm coupled (solid) and the $2 \times 10^{15} \mathrm{Ne} / \mathrm{cm}^{2} \mathrm{fm}$ coupled (dotted) sample. The MOKE reversal curves of the samples with intermediate fluences have the same basic shape as the as-deposited sample, meaning that they are all coupled afm. However, a monotonic decrease of both the saturation field $H_{\mathrm{s}}$ and the coupling field $H_{\mathrm{cp}}$ is observed with an increasing fluence. This corresponds to a continuous reduction of the afm IEC. The bilinear and biquadratic coupling constants for the afm IEC samples have been obtained by fitting an energy-minimized StonerWohlfarth model to the experimental curves as explained earlier.

For the fm IEC stacks the coupling constants were determined by FMR. Figure 4 shows the polar-angle-dependent FMR data for the single $\mathrm{Co}(25)$ and $\mathrm{NiFe}(25)$ reference samples, as well as for the fm coupled, ion-irradiated $\mathrm{Co} / \mathrm{Ru} / \mathrm{NiFe}(25)$ trilayer. The angle between the external field and the surface normal is denoted by $\theta_{\mathrm{H}}$. For both reference samples (Co: up-pointing triangle, NiFe: downpointing triangle) the uniform resonance mode is observed. Their resonance fields $H_{\text {res }}$ are plotted as a function of the external field orientation $\theta_{\mathrm{H}}$ in Fig. 4(a). For the irradiated $\mathrm{Co} / \mathrm{Ru} / \mathrm{NiFe}(25)$ trilayer two different modes are observed [see
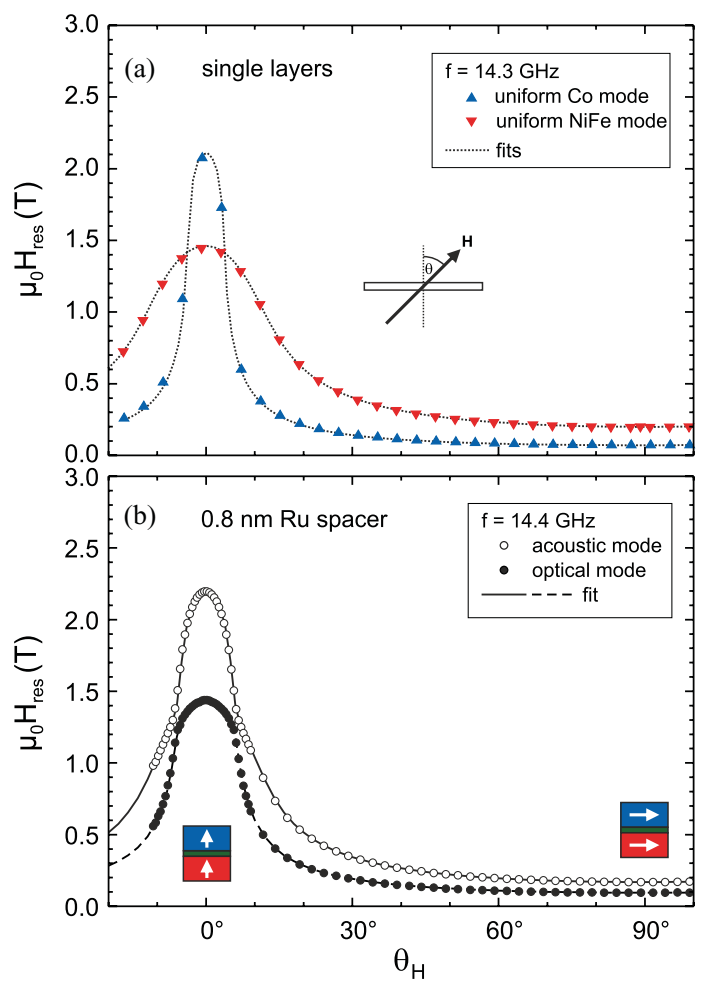

FIG. 4. (Color online) Polar angular dependencies of the resonance field of (a) the individual $\mathrm{Co}(25)$ and $\mathrm{NiFe}(25)$ reference films. (b) Acoustic and optical FMR resonance modes of the ionirradiated $\mathrm{Co} / \mathrm{Ru} / \mathrm{NiFe}(25)$ (fluence $2 \times 10^{15} \mathrm{Ne} / \mathrm{cm}^{2}$ ). The lines are fits according to the resonance equations in Ref. 38 .

Fig. 4(b)]. They correspond to an in-phase precession (acoustic mode, open circles) and an antiphase precession (optical mode, filled circles) of the two ferromagnetic layers, respectively. The distance of these two modes at the avoided cross point $\left(\theta_{\mathrm{H}}= \pm 6^{\circ}\right)$ scales with the fm coupling strength. The solid and dashed lines are fits according to the resonance equation given in Ref. 38. The fit yields a strong ferromagnetic bilinear coupling of $J_{\mathrm{L}}=0.7 \pm 0.1 \mathrm{~mJ} / \mathrm{m}^{2}$ for the $\mathrm{Co} / \mathrm{Ru} / \mathrm{NiFe}(25)$ trilayer irradiated with $2 \times 10^{15} \mathrm{Ne} / \mathrm{cm}^{2}$ and does not require any biquadratic coupling.

The values obtained for $J_{\mathrm{L}}$ and $J_{\mathrm{Q}}$ for the irradiated $\mathrm{Co} / \mathrm{Ru} / \mathrm{NiFe}(25)$ films are summarized in Table II. We observe a monotonic increase of both coupling constants with irradiation fluence, with $J_{\mathrm{L}}$ always being much larger than $J_{\mathrm{Q}}$. For the highest fluence $\left(2 \times 10^{15} \mathrm{Ne} / \mathrm{cm}^{2}\right) J_{\mathrm{L}}$ is positive and $J_{\mathrm{Q}}$ is zero. These results clearly show that ion irradiation with relatively low fluences yields the possibility to control the IEC magnitude and sign in magnetic multilayer films in a post-deposition process. A change from afm to fm coupling is induced beyond a certain fluence. For the $\mathrm{Co} / \mathrm{Ru} / \mathrm{NiFe}(25)$ film this transition fluence is estimated (by linear interpolation) to be $\sim 4 \times 10^{14} \mathrm{Ne} / \mathrm{cm}^{2}$ for $60 \mathrm{keV}$ irradiation. Within both coupling regimes, the bilinear coupling constant can be monotonously increased.

To understand the origin of the observed changes in the IEC, we have performed cross-sectional TEM measurements. These are shown in Fig. 5 for (a) an unirradiated sample and (b) a sample irradiated with $2 \times 10^{15} \mathrm{Ne} / \mathrm{cm}^{2}$. The unirradiated 

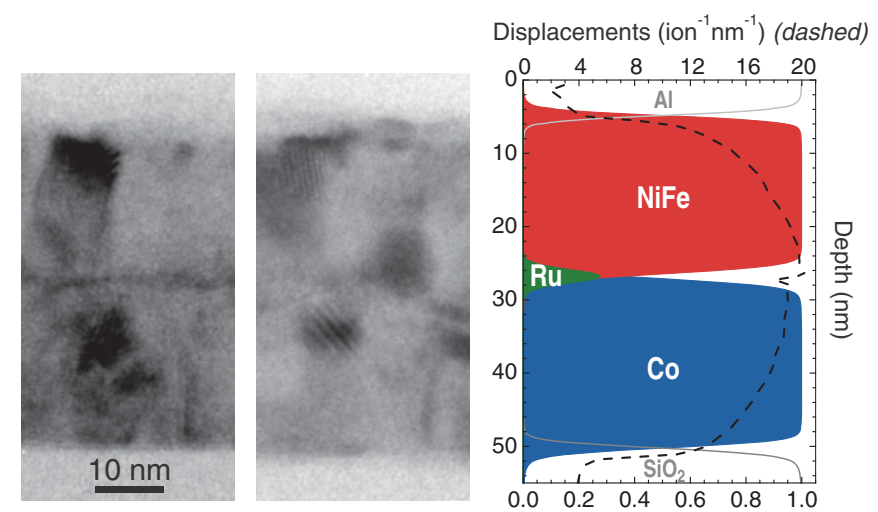

(a)

(b)

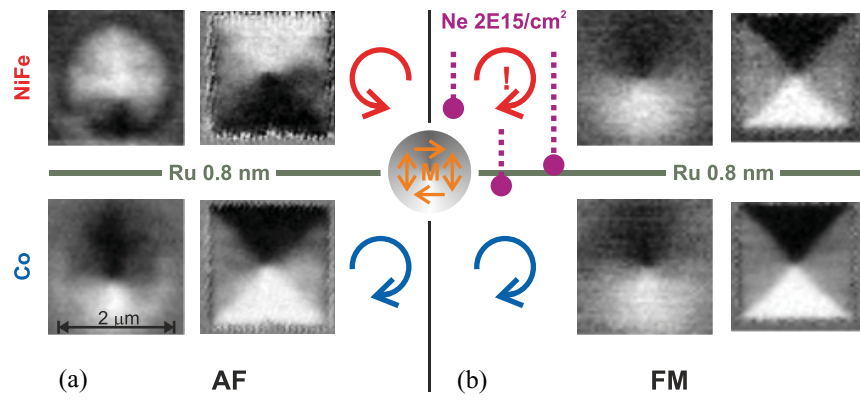

FIG. 6. (Color online) STXM images showing the layer-resolved magnetization patterns for the $\mathrm{NiFe}$ and Co layers in $\mathrm{Co} / \mathrm{Ru} / \mathrm{NiFe}(25)$ trilayer disks and squares. The as-prepared samples (a) show AF vortex pair configurations. Upon irradiation (b) they change to the FM state.

of $2 \mu \mathrm{m}$ size. The as-prepared structures exhibit the AF configuration (a). ${ }^{45}$ The NiFe layer shows a bright contrast in the upper half as opposed to a dark contrast in the lower half. This corresponds to a counterclockwise circulation $(c=1)$ as sketched to its right. The Co layer underneath shows the inverted intensities: Where $\mathrm{NiFe}$ is bright, $\mathrm{Co}$ is dark and vice versa. Thus, the Co layer exhibits a clockwise vortex circulation $(c=-1) .{ }^{46}$ The reason for the opposing circulations in this trilayer stack is the present afm IEC. Obviously the alignment angle $\left(\phi_{1}-\phi_{2}\right)$ is homogenous as has been shown previously. ${ }^{22}$ The interesting question is whether the homogeneity of the coupling is preserved when it is changed to fm IEC by ion irradiation. Figure 6(b) shows a trilayer disk and square after ion irradiation with $2 \times 10^{15} \mathrm{Ne} / \mathrm{cm}^{2}$. Note that the irradiated disks (squares) are from the same (a similar) fabrication batch as the unirradiated ones. In the irradiated structures dark $\mathrm{NiFe}$ regions are on top of dark Co regions and the bright regions are also on top of each other. Hence the vortices in both layers show clockwise circulations $\left(c_{\mathrm{Co}}=c_{\mathrm{NiFe}}=-1\right)$ as sketched. This means that FM configurations are exhibited. We observe undistorted flux closure patterns also for the irradiated samples. Although the $\mathrm{Ne}$ irradiation alters the sample morphology, the coupling angle remains laterally homogenous on a submicron scale. The ion-induced coupling reorientation shown is representative for the whole set of structures investigated.

Obviously, for both coupling orientations afm and fm, the control of IEC is sufficient to deterministically set the vortex pair circulation state. The reason for this is the relatively high IEC energy $\left(E_{\mathrm{IEC}}\right)$ that accounts for the whole sample area. Energy estimations show that for a $2 \mu \mathrm{m}$ structure the difference between the AF and the FM state is of the order of $10^{-15} \mathrm{~J}$. Compared to that, the demagnetization energy $\left(E_{\mathrm{dem}}\right)$ of $\sim 10^{-17} \mathrm{~J}$ is negligible, as it is only caused by the stray fields of the relatively small vortex cores.

Regarding the configuration of polarities however the situation is different. In the context of the relatively small areas relevant, the magnetodipolar core interaction becomes significant. Therefore an IEC-induced determination of the $P$ configuration cannot be generally expected. As a reliable imaging of the core polarities in vortex multilayers is beyond the experimental resolution available at present, we have addressed this point by micromagnetic simulations. A bilinear 


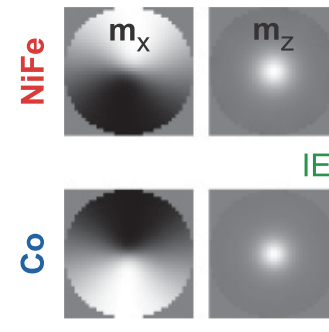

(a) $(\mathrm{AF}, \mathrm{PL})$
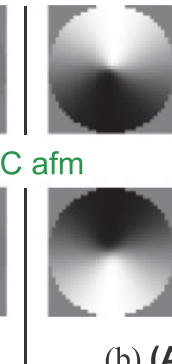

(b) (AF,AP)

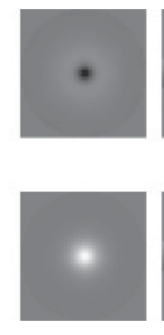

(c) $(\mathrm{FM}, \mathrm{AP})$

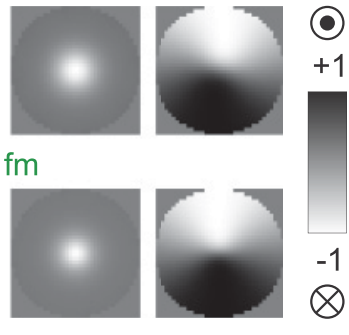

(d) $(\mathrm{FM}, \mathrm{PL})$

FIG. 7. (Color online) Micromagnetic simulations of vortex pair states in trilayer disks of $125 \mathrm{~nm}$ diameter. The magnetic orientation $\left(m_{x}, m_{z}\right)$ is displayed as indicated. (a), (b) AF circulation configurations for afm IEC. (c), (d) FM circulation states for fm IEC. The vortex cores correspond to the black or white dots in the $m_{z}$ contrast.

IEC can be either favoring the AP or the PL state depending on its sign. In contrast, the dipolar interaction always favors the PL configuration as long as the cores are on top of each other and their size is conserved. However, even for an fm IEC system, metastable AP states cannot be excluded when taking into account the very high fields necessary for quasistatic core reversals. ${ }^{47,48}$ Theoretically such reversals are actually forbidden in systems with continuous $M_{\mathrm{s}}$ due to the necessary formation of Bloch point singularities. ${ }^{19}$

In order to examine the possible core states we have performed three-dimensional micromagnetic simulations. ${ }^{49}$ Circular trilayer disks with $t$ and $M_{\mathrm{S}}$ values as measured have been modeled on a $36 \times 36 \times(5+1+5)$ grid..$^{50}$ In order to allow for an appropriate cell size, the lateral size was reduced to $125 \mathrm{~nm}$. Stationary states are accessed by integrating the Landau-Lifshitz-Gilbert equation ${ }^{51}$ with a high damping coefficient. For making the problem comparable-though not qualitatively changing the result- $J_{\mathrm{L}}$ was set to $\pm 0.5 \mathrm{~mJ} / \mathrm{m}^{2}$ while $J_{\mathrm{Q}}$ and the ion-induced $M_{\mathrm{s}}$ reduction were neglected. For each IEC orientation, both polarity states were initialized for the ground-state circulation configuration.

The simulations show that both polarity states, PL and AP, are stable for each coupling orientation. The PL configuration however is always about $8 \%$ lower in energy for the given coreto-total area ratio of $\sim 2.5 \%$. Figure 7 shows the calculated inplane $\left(m_{x}\right)$ and out-of-plane $\left(m_{z}\right)$ magnetization components close to the interlayer for the different cases. The panels (a), (b) and (c), (d) correspond to afm IEC and fm IEC, respectively. While PL states [Figs. 7(a) and 7(d)] are identified by dots of equal (white) contrast in the center of the $m_{z}$ images, AP states [Figs. 7(b) and 7(c)] exhibit an opposing black contrast in these regions. Interestingly, the simulated core radii are significantly smaller in the AP configurations. The average core radius (FWHM) is measured to $\bar{r}=(12 \pm 0.5) \mathrm{nm}$ for both cores ( $\mathrm{Co}$ and $\mathrm{NiFe}$ ) in the PL cases. In contrast, the core in the Co layer extends to $\bar{r}=(10 \pm 0.5) \mathrm{nm}$ with an NiFe counterpart of only $\bar{r}=(7 \pm 0.5) \mathrm{nm}$ and $\bar{r}=(5 \pm 0.5) \mathrm{nm}$ for afm AP and fm AP, respectively. The explanation for this effect is as follows. A virtual switching from PL to AP strongly increases $E_{\mathrm{dem}}$ as the core "dipoles" are pointing antiparallel now. The system reacts to that with a reduction in core size and thus in $E_{\text {dem }}$ while the layer internal exchange energy $E_{\text {ex }}$ slightly increases. ${ }^{25}$ The IEC then either reduces (afm) or increases (fm) the energy of the AP state, which reflects in the smallest NiFe core size for fm IEC.
The theoretical existence of both polarity configurations is in agreement with deductions from recently reported magnetoresistive experiments. ${ }^{29,30}$ At the given parameters, our simulations show that the PL state is the ground state for both IEC orientations. This can be understood by roughly estimating the relevant fields involved. The core stray fields are $\sim 1600 \mathrm{mT}(\mathrm{Co})$ and $\sim 930 \mathrm{mT}(\mathrm{NiFe})$, whereas the saturation field of the afm coupled IEC trilayers is only $\sim 50 \mathrm{mT}$. An increase of the afm IEC strength as well as a reduction of the ferromagnetic layer thicknesses could make the AP state the ground state of the afm branch. However, an IEC determination of the AP configuration seems to be out of reach. In contrast, for the fm branch such a determination of the PL state can be expected when higher ion fluences are used. It should be noted that the simulations do not account for sample inhomogeneities which could significantly reduce the barrier between PL and AP configurations.

\section{CONCLUSION}

We have investigated the ion-irradiation-induced modification of IEC regarding its global and micromagnetic effects. In agreement with previous works, ${ }^{13,14}$ a phase transition of the coupling from afm to $\mathrm{fm}$ in continuous films beyond a critical irradiation fluence of $\sim 4 \times 10^{-14} \mathrm{~cm}^{-2}$ was observed. Furthermore we provide a full quantitative evaluation of the coupling constants $\left(J_{\mathrm{L}}, J_{\mathrm{Q}}\right)$ by analyzing magnetization reversal loops and angle-dependent FMR curves. This evaluation reveals a continuous increase of the coupling constants from negative to positive values with increasing ion fluence. The reason for the IEC changes are ion-induced atomic collision processes and hence interfacial mixing. As shown by TEM imaging and ballistic simulations, the interfaces get smeared out significantly. This reduces the maximum relative concentration of interlayer atoms to below $1 / 3$. By these results we prove that the ion-induced IEC modification allows for a continuous and fine tuning of the coupling strength and orientation, while having a negligible impact on $M_{\mathrm{s}}$. As a post-deposition method it offers also a higher flexibility compared to the interlayer thickness variation.

The local magnetic coupling was evaluated for the model system of stacked vortex pairs in patterned trilayer disks and squares. The layer-specific magnetization orientation of the individual vortices has been imaged by means of STXM. We found the in-plane circulation configuration to be fully 
determined by the IEC orientation. Interestingly the in-plane alignment angle is homogenous throughout the whole structure for both cases. This means that for afm IEC the vortices are always in an AF state, while for fm IEC they are necessarily configured FM. According to micromagnetic simulations, IEC however is insufficient to enforce a corresponding vortex core alignment for the given $J$ values. Due to the very high coercivity of vortex cores, both states PL and AP are stable, independently from the IEC orientation. However, the relatively big core stray fields are always causing an energetic preference for the PL configuration. Interestingly, the vortex core sizes are a signature of the actual $P$ state, with AP having smaller core diameters than PL. A direct experimental observation of interlayer coupled vortex cores might become possible in the future by applying (S)TXM with ultrahigh resolution zone plates or by using samples with increased ferromagnetic layer thicknesses.

The results of this study provide further insight into the nature and control of IEC spin structures. The post-deposition modification of IEC by ion irradiation on the other hand is a promising tool for all fabrication processes where a fine control of IEC - even with lateral resolution - is desired. Also the possibility to determine the circulation state of interlayer coupled vortex pairs may have an impact on future vortexbased spin-transfer oscillators or memory cells.

\section{ACKNOWLEDGMENTS}

We would like to thank Aleksandar Puzić for his contribution to this work and the following people for their support at various stages: Vicki Kühn (lithography), Jochen Grebing and Kerstin Bernert (STXM), Julia Dschemuchadse and Julia Osten (reversal simulations), Arndt Mücklich and Monika Fritzsche (TEM), Stefan Eisenwinder and Udo Strauch (ion irradiation), and Wolfgang Michalke (sputter deposition). The STXM experiments were performed using the X07DA (PolLux) beamline at the Swiss Light Source, Paul Scherrer Institut, Villigen, Switzerland. Funding from BMBF (Contract No. 05 KS4WE1/6 and No. FKZ 13N10144) and EU (Grant No. 226716) is acknowledged. *s.wintz@hzdr.de

${ }^{\dagger}$ Present address: Vacuumschmelze GmbH \& Co. KG, 63450 Hanau, Germany.

${ }^{\ddagger}$ Present address: 01326 Dresden, Germany.

§Present address: Christian-Albrechts Universität zu Kiel, 24118 Kiel, Germany.

${ }^{1}$ M. N. Baibich, J. M. Broto, A. Fert, F. Nguyen Van Dau, F. Petroff, P. Etienne, G. Creuzet, A. Friederich, and J. Chazelas, Phys. Rev. Lett. 61, 2472 (1988).

${ }^{2}$ G. Binasch, P. Grünberg, F. Saurenbach, and W. Zinn, Phys. Rev. B 39, 4828 (1989).

${ }^{3}$ P. Grünberg, R. Schreiber, Y. Pang, M. B. Brodsky, and H. Sowers, Phys. Rev. Lett. 57, 2442 (1986).

${ }^{4}$ S. S. P. Parkin, R. Bhadra, and K. P. Roche, Phys. Rev. Lett. 66, 2152 (1991).

${ }^{5}$ P. Bruno, Phys. Rev. B 52, 411 (1995).

${ }^{6}$ S. S. P. Parkin, Phys. Rev. Lett. 67, 3598 (1991).

${ }^{7}$ M. Rührig, R. Schäfer, A. Hubert, R. Mosler, J. A. Wolf, S. Demokritov, and P. Grünberg, Phys. Status Solidi A 125, 635 (1991).

${ }^{8}$ J. Unguris, R. J. Celotta, and D. T. Pierce, Phys. Rev. Lett. 67, 140 (1991).

${ }^{9}$ J. Slonczewski, J. Magn. Magn. Mater. 150, 13 (1995).

${ }^{10}$ S. S. P. Parkin, N. More, and K. P. Roche, Phys. Rev. Lett. 64, 2304 (1990).

${ }^{11}$ C. Chappert, H. Bernas, J. Ferré, V. Kottler, J. P. Jamet, Y. Chen, E. Cambril, T. Devolder, F. Rousseaux, V. Mathet, and H. Launois, Science 280, 1919 (1998).

${ }^{12}$ J. Fassbender and J. McCord, J. Magn. Magn. Mater. 320, 579 (2008).

${ }^{13}$ S. O. Demokritov, C. Bayer, S. Poppe, M. Rickart, J. Fassbender, B. Hillebrands, D. I. Kholin, N. M. Kreines, and O. M. Liedke, Phys. Rev. Lett. 90, 097201 (2003).

${ }^{14}$ V. Höink, J. Schmalhorst, G. Reiss, T. Weis, D. Lengemann, D. Engel, and A. Ehresmann, J. Appl. Phys. 103, 123903 (2008).
${ }^{15}$ V. E. Demidov, D. I. Kholin, S. O. Demokritov, B. Hillebrands, F. Wegelin, and J. Marien, Appl. Phys. Lett. 84, 2853 (2004).

${ }^{16}$ S. Blomeier, B. Hillebrands, V. E. Demidov, S. O. Demokritov, B. Reuscher, A. Brodyanski, and M. Kopnarski, J. Appl. Phys. 98, 093503 (2005)

${ }^{17}$ S. Blomeier, P. Candeloro, B. Hillebrands, B. Reuscher, A. Brodyanski, and M. Kopnarski, Phys. Rev. B 74, 184405 (2006).

${ }^{18}$ J. Fassbender, L. Bischoff, R. Mattheis, and P. Fischer, J. Appl. Phys. 99, 08G301 (2006).

${ }^{19}$ A. Hubert and R. Schäfer, Magnetic Domains: The Analysis of Magnetic Microstructures (Springer, 2008).

${ }^{20}$ K. S. Buchanan, K. Y. Guslienko, S.-B. Choe, A. Doran, A. Scholl, S. D. Bader, and V. Novosad, J. Appl. Phys. 97, 10 H503 (2005).

${ }^{21}$ A. M. Kaiser, C. Schöppner, F. M. Römer, C. Hassel, C. Wiemann, S. Cramm, F. Nickel, P. Grychtol, C. Tieg, J. Lindner, and C. M. Schneider, Phys. Rev. B 84, 134406 (2011).

${ }^{22}$ S. Wintz, T. Strache, M. Körner, M. Fritzsche, D. Markó, I. Mönch, R. Mattheis, J. Raabe, C. Quitmann, J. McCord, A. Erbe, and J. Fassbender, Appl. Phys. Lett. 98, 232511 (2011).

${ }^{23}$ J. Wu, D. Carlton, E. Oelker, J. S. Park, E. Jin, E. Arenholz, A. Scholl, C. Hwang, J. Bokor, and Z. Q. Qiu, J. Phys.: Condens. Matter 22, 342001 (2010).

${ }^{24}$ C. Kittel, Rev. Mod. Phys. 21, 541 (1949).

${ }^{25}$ E. Feldtkeller and H. Thomas, Phys. Kondens. Materie 4, 8 (1965).

${ }^{26}$ R. P. Cowburn, D. K. Koltsov, A. O. Adeyeye, M. E. Welland, and D. M. Tricker, Phys. Rev. Lett. 83, 1042 (1999).

${ }^{27}$ T. Shinjo, T. Okuno, R. Hassdorf, K. Shigeto, and T. Ono, Science 289, 930 (2000).

${ }^{28}$ J. Raabe, R. Pulwey, R. Sattler, T. Schweinbök, J. Zweck, and D. Weiss, J. Appl. Phys. 88, 4437 (2000).

${ }^{29}$ S. S. Cherepov, A. Konovalenko, V. Korenivski, and D. C. Worledge, IEEE Trans. Magn. 46, 2124 (2010).

${ }^{30}$ N. Locatelli, V. V. Naletov, J. Grollier, G. de Loubens, V. Cros, C. Deranlot, C. Ulysse, G. Faini, O. Klein, and A. Fert, Appl. Phys. Lett. 98, 062501 (2011). 
${ }^{31}$ D. K. Schreiber, O. G. Heinonen, and A. K. Petford-Long, Phys. Rev. B 80, 014411 (2009).

${ }^{32}$ K. Y. Guslienko and K. L. Metlov, Phys. Rev. B 63, 100403(R) (2001).

${ }^{33}$ H. Hoffmann and F. Steinbauer, J. Appl. Phys. 92, 5463 (2002).

${ }^{34}$ K. L. Metlov and K. Y. Guslienko, J. Magn. Magn. Mater. 242-245, 1015 (2002).

${ }^{35}$ J. Biersack and L. Haggmark, Nucl. Instrum. Methods 174, 257 (1980)

${ }^{36}$ W. Möller and W. Eckstein, Nucl. Instrum. Methods Phys. Res. B 2, 814 (1984).

${ }^{37}$ D. Markó, T. Strache, K. Lenz, J. Fassbender, and R. Kaltofen, Appl. Phys. Lett. 96, 022503 (2010).

${ }^{38}$ J. Lindner and K. Baberschke, J. Phys.: Condens. Matter 15, S465 (2003).

${ }^{39}$ J. Lindner and K. Baberschke, J. Phys.: Condens. Matter 15, R193 (2003).

${ }^{40}$ J. Stöhr, Y. Wu, B. D. Hermsmeier, M. G. Samant, G. R. Harp, S. Koranda, D. Dunham, and B. P. Tonner, Science 259, 658 (1993).

${ }^{41}$ P. Fischer, G. Schütz, G. Schmahl, P. Guttmann, and D. Raasch, Z. Phys. B 101, 313 (1996).

${ }^{42}$ J. Raabe, G. Tzvetkov, U. Flechsig, M. Böge, A. Jaggi, B. Sarafimov, M. G. C. Vernooij, T. Huthwelker, H. Ade, D. Kilcoyne,
T. Tyliszczak, R. H. Fink, and C. Quitmann, Rev. Sci. Instrum. 79, 113704 (2008).

${ }^{43}$ M. Böge, U. Flechsig, J. Raabe, and T. Schilcher, in Proceedings of the Tenth European Particle Accelerator Conference EPAC 06, Edinburgh, Scotland (2006), p. 3610.

${ }^{44}$ G. Schütz, W. Wagner, W. Wilhelm, P. Kienle, R. Zeller, R. Frahm, and G. Materlik, Phys. Rev. Lett. 58, 737 (1987).

${ }^{45}$ Instead of a continuous curl square-shaped samples exhibit a Landau pattern, i.e., four discrete domains that are interconnected by $90^{\circ}$ domain walls.

${ }^{46}$ Note that the vortex cores in this specific AF disk are congruently displaced from the center. This is not a general effect (Ref. 22) but here presumably occurs due to pinning at sample defects.

${ }^{47}$ T. Okuno, K. Shigeto, T. Ono, K. Mibu, and T. Shinjo, J. Magn. Magn. Mater. 240, 1 (2002).

${ }^{48}$ A. Thiaville, J. M. García, R. Dittrich, J. Miltat, and T. Schrefl, Phys. Rev. B 67, 094410 (2003).

${ }^{49}$ M. R. Scheinfein, http://llgmicro.home.mindspring.com.

${ }^{50}$ Vacuum susceptibility was assumed for the interlayer. The exchange constants were set to $A_{\mathrm{Co}}=3.05 \times 10^{-11} \mathrm{~J} / \mathrm{m}$ and $A_{\mathrm{NiFe}}=1.05 \times$ $10^{-11} \mathrm{~J} / \mathrm{m}$.

${ }^{51}$ T. Gilbert, IEEE Trans. Magn. 40, 3443 (2004). 